\title{
Infinite Geraghty type extensions and their applications on integral equations
}

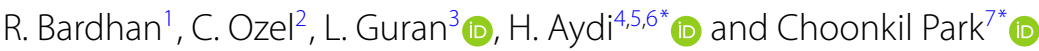

\author{
"Correspondence: \\ hassen.aydi@isima.rnu.tn; \\ baak@hanyang.ac.kr \\ ${ }^{4}$ Institut Supérieur d'Informatique et \\ des Techniques de Communication, \\ Université de Sousse, H. Sousse \\ 4000, Tunisia \\ 'Research Institute for Natural \\ Sciences, Hanyang University, Seoul \\ 04763, Korea \\ Full list of author information is \\ available at the end of the article
}

\begin{abstract}
In this article, we discuss about a series of infinite dimensional extensions of some theorems given in (Shumrani et al. in SER Math. Inform. 33(2):197-202, 2018), (Fisher in Math. Mag. 48(4):223-225, 1975), and (Fogh, Behnamian and Pashaie in Int. J. Maps in Mathematics 2(41):1-13, 2019). We also prove a similar Geraghty type construction for Fisher (Math. Mag. 48(4):223-225, 1975) in an infinite dimension using similar techniques as in (Shumrani et al. in SER Math. Inform. 33(2):197-202, 2018) and (Fogh, Behnamian and Pashaie in Int. J. Maps in Mathematics 2(41):1-13, 2019). As an application, we ensure the existence of solutions for infinite dimensional Fredholm integral equation and Uryshon type integral equation.
\end{abstract}

MSC: $47 \mathrm{H} 10 ; 54 \mathrm{H} 25 ; 45 \mathrm{A02} ; 45 \mathrm{~B} 02$

Keywords: Fixed point; Geraghty theorem; Complete metric space; Infinite dimension; $H_{k}$ contraction; $M_{k}$ function; $k$-dimensional extension

\section{Introduction and preliminaries}

The fixed point theory is an essential tool not only in the field of nonlinear analysis, but also in almost all branches of mathematics. After the Banach contraction principle [4], there were multiple generalizations that have been made. For instance, see [5-23]. Among these generalizations, we have Geraghty's theorem [24]. Moreover, in [1] a $k$-dimensional extension of Geraghty's result is also provided. Also, Kannan and Fisher gave two independent types of contraction operators (see $[2,25]$ ) which are completely independent of Banach's contraction. Also, in the recent paper [3], Fogh et al. introduced the concept of Kannan-Geraghty contractions.

In this paper, we extend the $k$-dimensional extension of Geraghty's theorem stated in [1] to an infinite dimension. We also introduce and develop a new notion of $H_{k}$ contractions for Geraghty-Kannan type operators from [3] to provide both $k$ dimensional extension and infinite dimensional extension. We also provide the same family of $k$-dimensional extensions for Fisher type contractions and also develop similar infinite dimensional extension in this case. As application, we define the notion of two independent types of infinite integral equations, namely infinite dimensional Fredholm equation and Uryshon type integral equation, and ensure the existence of solutions.

(c) The Author(s) 2021. This article is licensed under a Creative Commons Attribution 4.0 International License, which permits use sharing, adaptation, distribution and reproduction in any medium or format, as long as you give appropriate credit to the original author(s) and the source, provide a link to the Creative Commons licence, and indicate if changes were made. The images or other third party material in this article are included in the article's Creative Commons licence, unless indicated otherwise in a credit line to the material. If material is not included in the article's Creative Commons licence and your intended use is not permitted by statutory regulation or exceeds the permitted use, you will need to obtain permission directly from the copyright holder. To view a copy of this licence, visit http://creativecommons.org/licenses/by/4.0/. 
Next, we discuss some of the preliminaries which will be needed later for proving our main theorems. The following result is due to Geraghty [24].

Theorem 1.1 ([24]) Let $(X, d)$ be a complete metric space and $T: X \rightarrow X$ be a mapping such that

$$
d(T x, T y) \leq \beta(d(x, y)) d(x, y)
$$

where $\beta:[0, \infty) \rightarrow[0,1)$ is a function satisfying the condition

$$
\lim _{n \rightarrow \infty} \beta\left(t_{n}\right)=1 \text { implies } \quad \lim _{n \rightarrow \infty} t_{n}=0
$$

Then $T$ admits a unique fixed point $u \in X$ and $\left\{T^{n} x\right\}$ converges to $u$ for each $x \in X$.

We denote by $\mathbb{G}$ the set of all functions $\beta$ given in Theorem 1.1. We denote by $\mathbb{N}$ (resp. $\mathbb{N}_{0}$ ) the set of positive (nonnegative) integers.

Over the years, the study of a Geraghty type contraction has been the interest of many works in literature. This contraction was extended and generalized in many directions as Kannan-Geraghty type contractions, Fisher-Geraghty type contractions, etc. For more details, we refer the readers to [26-38].

Further, let us recall some results and definitions useful in our main results.

Theorem 1.2 ([25]) Let $(X, d)$ be a complete metric space and $T: X \rightarrow X$ be a mapping. If $T$ verifies

$$
d(T x, T y) \leq c(d(T x, x)+(T y, y))
$$

for all $x, y \in X$, where $c \in(0,1 / 2)$, then $T$ has a unique fixed point $u \in X$.

Theorem 1.3 [2] Let $(X, d)$ be a complete metric space and $T: X \rightarrow X$ be a mapping. If $T$ verifies

$$
d(T x, T y) \leq c(d(T x, y)+d(T y, x))
$$

for all $x, y \in X$, where $c \in(0,1 / 2)$, then $T$ possesses a unique fixed point $u \in X$.

Definition 1.1 (Kannan-Geraghty map, see [3]) Let $T$ be a self-map on a metric space $(X, d)$. Such $T$ is said to be a Kannan-Geraghty contraction if and only if it satisfies the following:

$$
d(T x, T y) \leq \frac{\beta(d(x, y))}{2}(d(T x, x)+d(T y, y))
$$

for all $x, y \in X$, where $\beta \in \mathbb{G}$.

Theorem 1.4 (see [3]) Let $(X, d)$ be a complete metric space and $T: X \rightarrow X$ be a mapping. If $T$ is a Kannan-Geraghty contraction on $(X, d)$, then $T$ possesses a unique fixed point $u \in X$, and for any $x_{0} \in X,\left\{T^{n} x_{0}\right\}$ converges to $u$. 
Theorem 1.5 ([1]) Let $(X, d)$ be a complete metric space and $k \in \mathbb{N}$. Let $T: X^{k} \rightarrow X$ be such that

$$
\begin{aligned}
& d\left(T\left(x_{1}, \ldots, x_{k}\right), T\left(x_{2}, \ldots, x_{k+1}\right)\right) \\
& \left.\quad \leq M\left(\left(x_{1}, \ldots, x_{k}\right),\left(x_{2}, \ldots, x_{k+1}\right)\right)\right) M\left(\left(x_{1}, \ldots, x_{k}\right),\left(x_{2}, \ldots, x_{k+1}\right)\right),
\end{aligned}
$$

for all $x_{1}, x_{2}, \ldots, x_{k+1} \in X$, where $\beta \in \mathbb{G}$ and $M: X^{k} \times X^{k} \rightarrow[0, \infty)$ is given as

$$
\begin{aligned}
& M\left(\left(x_{1}, \ldots, x_{k}\right),\left(x_{2}, \ldots, x_{k+1}\right)\right) \\
& \quad=\max \left\{d\left(x_{k}, x_{k+1}\right),\left(x_{k}, T\left(x_{1}, \ldots, x_{k}\right)\right), d\left(x_{k+1}, T\left(x_{2}, \ldots, x_{k+1}\right)\right)\right\} .
\end{aligned}
$$

Then there exists a point $u \in X$ such that $T(u, u, \ldots, u)=u$.

The aim of the next section is to generalize and extend Theorem 1.1, Theorem 1.4, and Theorem 1.5 as well as the $k$-dimensional extension of the result given in [24] to an infinite dimension. We denote the infinite tuples of points $\left(x_{1}, x_{2}, \ldots\right)$ by $\left(x_{k}\right)_{i=1}^{\infty}$ and the infinite tuples $\left(x_{1}, x_{2}, \ldots, x_{k-1}, x_{k}, x_{k}, \ldots\right)$ with the $k$ th point repeated by $\left(x_{j, \hat{k}}\right)_{j=1}^{\infty}$.

\section{Main results}

From now on, let us consider that $(X, d)$ is a complete metric space and $k \in \mathbb{N}$.

Definition 2.1 (Extended $M_{k}$ function) For any $\left(u_{i}\right)_{i=1}^{\infty},\left(v_{i}\right)_{i=1}^{\infty} \in \prod_{i=1}^{\infty} X$ and $T: \prod_{i=1}^{\infty} X \rightarrow$ $X$, we define the extended function $M_{k}: \prod_{l=1}^{\infty} X \times \prod_{l=1}^{\infty} X \rightarrow X$ as follows:

$$
\begin{aligned}
& M_{k}\left(\left(u_{i}\right)_{i=1}^{\infty},\left(v_{i}\right)_{i=1}^{\infty}\right) \\
& \quad=\left\{\begin{array}{l}
\max \left\{\sup _{l \geq k} d\left(u_{l}, v_{l}\right), \sup _{l \geq k} d\left(u_{l}, T\left(u_{i, \hat{l}}\right)_{i=1}^{\infty}\right), \sup _{l \geq k} d\left(v_{l}, T\left(v_{i, \hat{l}}\right)_{i=1}^{\infty}\right)\right\}, \\
\quad \text { if all supremum exists } \\
\max \left\{d\left(u_{k}, v_{k}\right), d\left(u_{k}, T\left(u_{i, \hat{k}}\right)_{i=1}^{\infty}\right), d\left(v_{k}, T\left(v_{i, \hat{k}}\right)_{i=1}^{\infty}\right)\right\}, \\
\quad \text { if one fails to exist }
\end{array}\right.
\end{aligned}
$$

Example 2.1 Consider $X=[0,1]$. For $k=1$, take the operator $T: \prod_{i=1}^{\infty} X \rightarrow X$ defined by $T\left(\left(u_{i}\right)_{i=1}^{\infty}\right)=u_{k}=u_{1}$ and $M_{k}\left(\left(u_{i}\right)_{i=1}^{\infty},\left(v_{i}\right)_{i=1}^{\infty}\right)=\left|u_{k}-v_{k}\right|=\left|u_{1}-v_{1}\right|$.

Definition 2.2 $\left(H_{k}\right.$ contraction) An operator $T: \prod_{l=1}^{\infty} X \rightarrow X$ is called an $H_{k}$ contraction if and only if it satisfies the following inequality:

$$
d\left(T\left(\left(u_{i, \widehat{k}}\right)_{i=1}^{\infty}\right), T\left(\left(v_{i, \widehat{k}}\right)_{i=1}^{\infty}\right)\right) \leq \beta\left(M_{k}\left(\left(u_{i, \widehat{k}}\right)_{i=1}^{\infty},\left(v_{i, \widehat{k}}\right)_{i=1}^{\infty}\right)\right) M_{k}\left(\left(u_{i, \hat{k}}\right)_{i=1}^{\infty},\left(v_{i, \widehat{k}}\right)_{i=1}^{\infty}\right)
$$

for all $u_{1}, u_{2}, \ldots, u_{k}, v_{1}, v_{2}, \ldots, v_{k} \in X$, where $\beta \in \mathbb{G}$.

Example 2.2 Consider $X=[0,1]$. The operator $T: \prod_{i=1}^{\infty} X \rightarrow X$ defined by $T\left(\left(x_{i}\right)_{i=1}^{\infty}\right):=c x_{k}$ is an $H_{k}$ contraction, where $k \in \mathbb{N}$ is any fixed number and $c \in(0,1)$.

Definition 2.3 (Kannan-Geraghty $H_{k}$ contraction) An operator $T: \prod_{l=1}^{k} X \rightarrow X$ is called a Kannan-Geraghty contraction of dimension $k$ if and only if it satisfies the following 
inequality:

$$
d\left(T\left(\left(u_{i}\right)_{i=1}^{k}\right), T\left(\left(v_{i}\right)_{i=1}^{k}\right)\right) \leq \frac{\beta\left(d\left(u_{k}, v_{k}\right)\right)}{2}\left(d\left(T\left(\left(u_{i}\right)_{i=1}^{k}\right), u_{k}\right)+d\left(T\left(\left(v_{i}\right)_{i=1}^{k}\right), v_{k}\right)\right)
$$

for all $u_{1}, u_{2}, \ldots, u_{k}, v_{1}, v_{2}, \ldots, v_{k} \in X$, where $\beta \in \mathbb{G}$.

Definition 2.4 (Extended Kannan-Geraghty $H_{k}$ contraction) An operator $T: \prod_{l=1}^{\infty} X \rightarrow$ $X$ is called an extended Kannan-Geraghty $H_{k}$ contraction if and only if it satisfies the following:

$$
d\left(T\left(\left(u_{i, \widehat{k}}\right)_{i=1}^{\infty}\right), T\left(\left(v_{i, \widehat{k}}\right)_{i=1}^{\infty}\right)\right) \leq \frac{\beta\left(d\left(u_{k}, v_{k}\right)\right)}{2}\left(d\left(T\left(\left(u_{i, \widehat{k}}\right)_{i=1}^{\infty}\right), u_{k}\right)+d\left(T\left(\left(v_{i, \widehat{k}}\right)_{i=1}^{\infty}\right), v_{k}\right)\right)
$$

for all $u_{1}, u_{2}, \ldots, u_{k}, v_{1}, v_{2}, \ldots, v_{k} \in X$, where $\beta \in \mathbb{G}$.

Definition 2.5 (Fisher-Geraghty $H_{k}$ contraction) An operator $T: \prod_{l=1}^{k} X \rightarrow X$ is called a Fisher-Geraghty $H_{k}$ contraction of dimension $k$ if and only if it satisfies the following:

$$
d\left(T\left(\left(u_{i}\right)_{i=1}^{k}\right), T\left(\left(v_{i}\right)_{i=1}^{k}\right)\right) \leq \frac{\beta\left(d\left(u_{k}, v_{k}\right)\right)}{2}\left(d\left(T\left(\left(u_{i}\right)_{i=1}^{k}\right), v_{k}\right)+d\left(T\left(\left(v_{i}\right)_{i=1}^{k}\right), u_{k}\right)\right)
$$

for all $u_{1}, u_{2}, \ldots, u_{k}, v_{1}, v_{2}, \ldots, v_{k} \in X$, where $\beta \in \mathbb{G}$.

Definition 2.6 (Extended Fisher-Geraghty $H_{k}$ contraction) An operator $T: \prod_{l=1}^{\infty} X \rightarrow$ $X$ is called an extended Kannan-Geraghty $H_{k}$ contraction if and only if it satisfies the following:

$$
d\left(T\left(\left(u_{i, \widehat{k}}\right)_{i=1}^{\infty}\right), T\left(\left(v_{i, \hat{k}}\right)_{i=1}^{\infty}\right)\right) \leq \frac{\beta\left(d\left(u_{k}, v_{k}\right)\right)}{2}\left(d\left(T\left(\left(u_{i, \widehat{k}}\right)_{i=1}^{\infty}\right), v_{k}\right)+d\left(T\left(\left(v_{i, \widehat{k}}\right)_{i=1}^{\infty}\right), u_{k}\right)\right)
$$

for all $u_{1}, u_{2}, \ldots, u_{k}, v_{1}, v_{2}, \ldots, v_{k} \in X$, where $\beta \in \mathbb{G}$.

Example 2.3 As an example for Definition 2.3 and Definition 2.5, we can consider, for $X=[0,1]$, the operator $T: \prod_{i=1}^{k} X \rightarrow X$ defined by $T\left(\left(x_{i}\right)_{i=1}^{k}\right)=c x_{k}$ for some $c \in\left(0, \frac{1}{2}\right)$.

Example 2.4 As an example for Definition 2.4 and Definition 2.6, for $X=[0,1]$, take the operator $T: \prod_{i=1}^{\infty} X \rightarrow X$ defined by $T\left(\left(x_{i}\right)_{i=1}^{\infty}\right)=c x_{k}$ for some $c \in\left(0, \frac{1}{2}\right)$.

The theory of Picard operators was developed by Rus and is used a lot in proving the existence and uniqueness of a solution of different types of integral or differential equations. For more details, see $[39,40]$.

Definition 2.7 (Picard operator) Let $(X, d)$ be a metric space. An operator $T: X \rightarrow X$ is a Picard operator if there exists $x^{*} \in X$ such that FixT $=\left\{x^{*}\right\}$ and the sequence $\left(T^{n}\left(x_{0}\right)\right)_{n \in \mathbb{N}}$ converges to $x^{*}$ for all $x_{0} \in X$.

Inspired by the Picard operator definition, we extend this notion for the $k$-dimensional and infinite dimensional cases as follows. 
Definition 2.8 ( $k$-Picard sequence with respect to the operator $T$ ) Let $T: \prod_{i=1}^{k} X \rightarrow X$ be any operator, and let us choose $x_{1}, x_{2}, \ldots, x_{k} \in X$. The $k$-Picard sequence with respect to the operator $T$ based on the base point set $\left\{x_{1}, x_{2}, \ldots, x_{k}\right\}$ is defined as $x_{n+k}:=T\left(\left(x_{n+i-1}\right)_{i=1}^{k}\right)$ for all $n \geq k$.

Example 2.5 If we fix $k=1$, then the base point set is singleton and the 1-Picard sequence with respect to $T$ based on $\left\{x_{0}\right\}$ is basically the Picard sequence of $T$ based on the base point $\left\{x_{0}\right\}$ defined by $x_{n}:=T\left(x_{n-1}\right)$ for all $n \geq 1$ and some $x_{0} \in X$.

Now, we define the following notions.

Definition 2.9 (Infinite $k$-Picard sequence with respect to the operator $T$ ) Let $T$ : $\prod_{i=1}^{\infty} X \rightarrow X$ be any operator, and let us choose $x_{1}, x_{2}, \ldots, x_{k}, \ldots \in X$. The $k$-Picard sequence with respect to the operator $T$ based on the base point set $\left\{x_{1}, x_{2}, \ldots, x_{k}, \ldots\right\}$ is defined as $x_{n+k}:=T\left(\left(x_{n+i-1, \widehat{n+k-1}}\right)_{i=1}^{\infty}\right)$ for all $n \geq k$.

Example 2.6 If we fix $k=1$, then the base point set is singleton and the infinite 1- Picard sequence with respect to $T$ based on the base point $\left\{x_{0}\right\}$ is basically the sequence defined by $x_{n}:=T\left(\left(x_{n-1}\right)_{n=1}^{\infty}\right) \forall n \geq 1$ for some $x_{0} \in X$.

Let us give our first main fixed point result, which is a generalization of the Banach contraction principle with respect to the infinite-dimensional notion introduced in our paper.

Theorem 2.1 Let $(X, d)$ be a complete metric space. $T: \prod_{l=1}^{\infty} X \rightarrow X$ is an $H_{k}$ contraction for some $k \in \mathbb{N}$. Then there exists $u \in X$ such that $T\left((u)_{i=1}^{\infty}\right)=u$ and the infinite $k$-Picard sequence for $T$ converges to $u$.

Proof Let $x_{1}, x_{2} \ldots, x_{k} \in X$. Then an infinite $k$-Picard sequence is defined as follows: $\forall n \in \mathbb{N}$ we define

$$
x_{n+k}:=T\left(\left(x_{n+i-1, \widehat{n+k-1}}\right)_{i=1}^{\infty}\right) .
$$

We claim that $\left\{d\left(x_{n+k}, x_{n+k+1}\right)\right\}_{n \in \mathbb{N}}$ is convergent. From Definition 2.2, one writes

$$
\begin{aligned}
& d\left(x_{n+k+1}, x_{n+k+2}\right)=d\left(T\left(\left(x_{n+i, \widehat{n+k}}\right)_{i=1}^{\infty}\right), T\left(\left(x_{n+i+1, \widehat{n+1+k}}\right)_{i=1}^{\infty}\right)\right) \\
& \quad \leq \beta\left(M_{k}\left(\left(x_{n+i, \widehat{n+k}}\right)_{i=1}^{\infty},\left(x_{n+1+i, \widehat{n+1+k}}\right)_{i=1}^{\infty}\right)\right) M_{k}\left(\left(x_{n+i, \widehat{n+k}}\right)_{i=1}^{\infty},\left(x_{n+1+i, \widehat{n+1+k}}\right)_{i=1}^{\infty}\right) .
\end{aligned}
$$

Let

$$
\begin{aligned}
& M_{k}\left(\left(x_{n+i, \widehat{n+k}}\right)_{i=1}^{\infty},\left(x_{n+i+1, \widehat{n+1+k}}\right)_{i=1}^{\infty}\right) \\
& \quad=\max \left\{\sup _{l \geq k} d\left(u_{l}, v_{l}\right), \sup _{l \geq k} d\left(u_{l}, T\left(u_{i, \widehat{l}}\right)_{i=1}^{\infty}\right), \sup _{l \geq k} d\left(v_{l}, T\left(v_{i, \hat{l}}\right)_{i=1}^{\infty}\right)\right\},
\end{aligned}
$$

where

$$
u_{l}:=x_{n+l}, \quad \forall 1 \leq l \leq k,
$$




$$
\begin{aligned}
& u_{l}:=x_{n+k}, \quad \forall l>k, \\
& v_{l}:=x_{n+l+1}, \quad \forall 1 \leq l \leq k, \\
& u_{l}:=x_{n+k+1}, \quad \forall l>k .
\end{aligned}
$$

But

$$
\begin{aligned}
& \sup _{l \geq k} d\left(\left(u_{l}, v_{l}\right)=d\left(\left(x_{n+k}, x_{n+k+1}\right),\right.\right. \\
& \sup _{l \geq k} d\left(\left(u_{l}, T\left(\left(u_{i, \widehat{l}}\right)_{i=1}^{\infty}\right)\right)=d\left(\left(x_{n+k}, T\left(\left(x_{n+i, \widehat{n+k}}\right)_{i=1}^{\infty}\right)\right),\right.\right. \\
& \sup _{l \geq k} d\left(\left(v_{l}, T\left(\left(v_{i, \hat{l}}\right)_{i=1}^{\infty}\right)\right)=d\left(\left(x_{n+k+1}, T\left(\left(x_{n+i+1, \widehat{n+k+1}}\right)_{i=1}^{\infty}\right)\right) .\right.\right.
\end{aligned}
$$

We have

$$
\begin{aligned}
& M_{k}\left(\left(x_{n+i, \widehat{n+k}}\right)_{i=1}^{\infty},\left(x_{n+i+1, \widehat{n+k+1}}\right)_{i=1}^{\infty}\right) \\
& \quad=\max \left\{d \left(\left(x_{n+k}, x_{n+k+1}\right), d\left(\left(x_{n+k}, T\left(\left(x_{n+i, \widehat{n+k}}\right)_{i=1}^{\infty}\right)\right), d\left(\left(x_{n+k+1}, T\left(\left(x_{n+1+i, \widehat{n+k+1}}\right)_{i=1}^{\infty}\right)\right)\right\} .\right.\right.\right.
\end{aligned}
$$

That is,

$$
\begin{aligned}
& M_{k}\left(\left(x_{n+i, n+k}\right)_{i=1}^{\infty},\left(x_{n+1+i, \widehat{n+k+1}}\right)_{i=1}^{\infty}\right) \\
& \quad=\max \left\{d \left(\left(x_{n+k}, x_{n+k+1}\right), d\left(\left(x_{n+k}, x_{n+k+1}\right), d\left(\left(x_{n+k+1}, x_{n+k+2}\right)\right\}\right.\right.\right. \\
& \quad=\max \left\{d \left(\left(d_{n+k}, d_{n+k+1}\right), d\left(\left(x_{n+k+1}, x_{n+k+2}\right)\right\} .\right.\right.
\end{aligned}
$$

Without loss of generality, assume that $d\left(\left(x_{n+k+1}, x_{n+k+2}\right)>0\right.$ for each $k$.

If, for some $k$, we have $M_{k}\left(\left(x_{n+i, \widehat{n+k}}\right)_{i=1}^{\infty},\left(x_{n+i+1, \widehat{n+k+1}}\right)_{i=1}^{\infty}\right)=d\left(\left(x_{n+k+1}, x_{n+k+2}\right)\right.$, then, from Definition 2.2, we get

$$
\begin{aligned}
0 & <d\left(\left(x_{n+k+1}, x_{n+k+2}\right)=d\left(\left(T\left(\left(x_{n+i, \widehat{n+k}}\right)_{i=1}^{\infty}\right), T\left(\left(x_{n+i+1, \widehat{n+k+1}}\right)_{i=1}^{\infty}\right)\right)\right.\right. \\
& \leq \beta\left(M_{k}\left(\left(x_{n+i, \widehat{n+k}}\right)_{i=1}^{\infty},\left(x_{n+i+1, \widehat{n+k+1}}\right)_{i=1}^{\infty}\right)\right) M_{k}\left(\left(x_{n+i, n+k}\right)_{i=1}^{\infty},\left(x_{n+i+1, \widehat{n+k+1}}\right)_{i=1}^{\infty}\right) \\
& =\beta\left(d ( ( x _ { n + k + 1 } , x _ { n + k + 2 } ) ) d \left(\left(x_{n+k+1}, x_{n+k+2}\right)\right.\right. \\
& <d\left(\left(x_{n+k+1}, x_{n+k+2}\right) .\right.
\end{aligned}
$$

Then we get a contradiction. Hence, for each $k, M_{k}\left(\left(x_{n+i, n+k}\right)_{i=1}^{\infty},\left(x_{n+i+1, \widehat{n+k+1}}\right)_{i=1}^{\infty}\right)=$ $d\left(\left(x_{n+k}, x_{n+k+1}\right)\right.$. One writes

$$
d\left(\left(x_{n+k+1}, x_{n+k+2}\right) \leq \beta\left(d ( ( x _ { n + k } , x _ { n + k + 1 } ) ) d \left(\left(x_{n+k}, x_{n+k+1}\right)<d\left(\left(x_{n+k}, x_{n+k+1}\right) .\right.\right.\right.\right.
$$

We deduce that $\left\{x_{n+k}\right\}_{n \in \mathbb{N}}$ is a strictly decreasing sequence. So there is $(r \geq 0) \in \mathbb{R}$ so that

$$
\lim _{n \rightarrow \infty} d\left(x_{n+k}, x_{n+k+1}\right)=r
$$

We claim $\lim _{n \rightarrow \infty} d\left(x_{n+k}, x_{n+k+1}\right)=0$. Suppose on the contrary that $r>0$. We have

$$
d\left(\left(x_{n+k+1}, x_{n+k+2}\right) \leq \beta\left(d ( ( x _ { n + k } , x _ { n + k + 1 } ) ) d \left(\left(x_{n+k}, x_{n+k+1}\right) .\right.\right.\right.
$$


That is,

$$
\frac{d\left(\left(x_{n+k+1}, x_{n+k+2}\right)\right.}{d\left(\left(x_{n+k}, x_{n+k+1}\right)\right.} \leq \beta\left(d\left(\left(x_{n+k}, x_{n+k+1}\right)\right) .\right.
$$

This implies that $\lim _{n \rightarrow \infty} \beta\left(d\left(\left(x_{n+k}, x_{n+k+1}\right)\right) \geq 1\right.$.

Since $\beta \in \mathbb{G}$, necessarily $\lim _{n \rightarrow \infty} \beta\left(d\left(\left(x_{n+k}, x_{n+k+1}\right)\right)=1\right.$, and so

$$
\lim _{n \rightarrow \infty} d\left(\left(x_{n+k}, x_{n+k+1}\right)=0 .\right.
$$

We now claim that $\left\{x_{n+k}\right\}_{n \in \mathbb{N}}$ is Cauchy, and we prove it by contradiction. Suppose on the contrary that there is $\varepsilon>0$ such that we can find some subsequences $\left\{x_{m(q)+k}\right\}_{p \in \mathbb{N}}$, $\left\{x_{n(q)+k}\right\}_{p \in \mathbb{N}}$ with $m(q)>n(q)>q$ such that, for every $q$, we have

$$
d\left(\left(x_{m(q)+k}, x_{n(q)+k}\right) \geq \varepsilon .\right.
$$

Moreover, corresponding to each $n(q)$, we can choose least of such $m(q)$ satisfying (8). Then

$$
d\left(\left(x_{m(q)+k-1}, x_{n(q)+k}\right)<\varepsilon .\right.
$$

From (7),(9) and using the triangle inequality, we get

$$
\begin{aligned}
d\left(\left(x_{m(q)+k-1}, x_{n(q)+k-1}\right)\right. & \leq d\left(\left(x_{m(q)+k-1}, x_{n(q)+k}\right)+d\left(\left(x_{n(q)+k-1}, x_{n(q)+k}\right)\right.\right. \\
& <\varepsilon+d\left(\left(x_{n(q)+k-1}, x_{n(q)+k}\right)\right.
\end{aligned}
$$

and

$$
\begin{aligned}
\varepsilon & \leq d\left(\left(x_{n(q)+k}, x_{m(q)+k}\right)\right. \\
& \leq d\left(\left(x_{n(q)+k}, x_{n(q)+k-1}\right)+d\left(\left(x_{n(q)+k-1}, x_{m(q)+k-1}\right)+d\left(\left(x_{m(q)+k-1}, x_{m(q)+k}\right) .\right.\right.\right.
\end{aligned}
$$

Letting $q \rightarrow \infty$ in (10) and using (11), we get

$$
\lim _{q \rightarrow \infty} d\left(\left(x_{m(q)+k-1}, x_{n(q)+k-1}\right)=\varepsilon\right.
$$

On the other hand, if

$$
\begin{aligned}
& M_{k}\left(\left(x_{n(q)+i-1, n(q)+k-1}\right)_{i=1}^{\infty},\left(x_{m(q)+i-1, m(q)+k-1}\right)_{i=1}^{\infty}\right) \\
& \quad=\max \left\{\operatorname { s u p } _ { l \geq k - 1 } d \left(\left(u_{l}, v_{l}\right), \sup _{l \geq k-1} d\left(\left(u_{l}, T\left(\left(u_{i, \widehat{l}}\right)_{i=1}^{\infty}\right)\right), \sup _{l \geq k-1} d\left(\left(v_{l}, T\left(\left(v_{i, \hat{l}}\right)_{i=1}^{\infty}\right)\right)\right\},\right.\right.\right.
\end{aligned}
$$

where

$$
\begin{aligned}
& u_{l}:=x_{n(q)+l-1}, \quad \forall 1 \leq l \leq k, \\
& u_{l}:=x_{n(q)+k-1}, \quad \forall l>k,
\end{aligned}
$$




$$
\begin{array}{ll}
v_{l}:=x_{m(q)+l-1}, & \forall 1 \leq l \leq k, \\
u_{l}:=x_{m(q)+k-1}, & \forall l>k
\end{array}
$$

then

$$
\begin{aligned}
& M_{k}\left(\left(x_{n(q)+i-1, n} \widehat{(q)+k-1}\right)_{i=1}^{\infty},\left(x_{m(q)+i-1, m} \widehat{(q)+k-1}\right)_{i=1}^{\infty}\right) \\
& \quad=\max \left\{\xi \left(\left(x_{n(q)+k-1}, x_{m(q)+k-1}\right), d\left(\left(x_{n(q)+k-1}, T\left(\left(x_{n(q)+i-1, n} \widehat{(q)+k-1}\right)_{i=1}^{\infty}\right)\right),\right.\right.\right. \\
& \left.d\left(\left(x_{m(q)+k-1}, T\left(\left(x_{m(q)+i-1, m(q)+k-1}\right)_{i=1}^{\infty}\right)\right)\right)\right\} \\
& \quad=\max \left\{d\left(\left(x_{n(q)+k-1}, x_{m(q)+k-1}\right), d\left(x_{n(q)+k-1}, x_{n(q)+k}\right), d\left(x_{m(q)+k-1}, x_{m(q)+k}\right)\right\} .\right.
\end{aligned}
$$

Using (7) and (12) we get

$$
\lim _{q \rightarrow \infty} M_{k}\left(\left(x_{n(q)+i-1, n \widehat{(q)+k-1}}\right)_{i=1}^{\infty},\left(x_{m(q)+i-1, m(\widehat{q)+k-1}}\right)_{i=1}^{\infty}\right)=\varepsilon
$$

By (1) and (17) we get

$$
\begin{aligned}
\varepsilon \leq & d\left(\left(x_{n(q)+k}, x_{m(q)+k}\right)=d\left(\left(T\left(x_{n(q)+i-1, n \widehat{(q)+k-1}}\right)_{i=1}^{\infty}, T\left(x_{m(q)+i-1, m} \widehat{m(q)+k-1}\right)_{i=1}^{\infty}\right)\right)\right. \\
\leq & \beta\left(M_{k}\left(\left(x_{n(q)+i-1, n \widehat{(q)+k}-1}\right)_{i=1}^{\infty},\left(x_{m(q)+i-1, m \widehat{(q)+k}-1}\right)_{i=1}^{\infty}\right)\right) \\
& \times M_{k}\left(\left(x_{n(q)+i-1, n \widehat{(q)+k}-1}\right)_{i=0}^{\infty},\left(x_{m(q)+i-1, m \widehat{(q)+k}-1}\right)_{i=1}^{\infty}\right) \\
< & M_{k}\left(\left(x_{n(q)+i-1, n \widehat{(q)+k-1}}\right)_{i=1}^{\infty},\left(x_{m(q)+i-1, m \widehat{(q)+k}-1}\right)_{i=1}^{\infty}\right) .
\end{aligned}
$$

\section{If we suppose}

$$
\begin{aligned}
& \sup _{l \geq k} d\left(\left(u_{l}, v_{l}\right)=d\left(\left(x_{n(q)+k-1}, x_{m(q)+k-1}\right),\right.\right. \\
& \sup _{l \geq k} d\left(\left(u_{l}, T\left(u_{i, \widehat{l}}\right)_{i=1}^{\infty}\right)=d\left(\left(x_{n(q)+k-1}, T\left(\left(x_{n(q)+i-1, n(q)+k-1}\right)_{i=1}^{\infty}\right)\right),\right.\right. \\
& \sup _{l \geq k} d\left(\left(v_{l}, T\left(v_{i, \widehat{l}}\right)_{i=1}^{\infty}\right)=d\left(\left(x_{m(q)+k-1}, T\left(\left(x_{m(q)+i-1, m(\overline{q)+k-1}}\right)_{i=1}^{\infty}\right)\right),\right.\right.
\end{aligned}
$$

then

$$
\begin{aligned}
& M_{k}\left(\left(x_{n(q)+i-1, n \widehat{(q)+k-1}}\right)_{i=1}^{\infty},\left(x_{m(q)+i-1, \overline{m(q)+k-1}}\right)_{i=1}^{\infty}\right) \\
& \quad=\max \left\{d \left(\left(x_{n(q)+k}, x_{m(q)+k}\right), d\left(\left(x_{n(q)+k-1}, T\left(\left(x_{n(q)+i-1, n(q)+k-1}\right)_{i=1}^{\infty}\right)\right),\right.\right.\right. \\
& \left.d\left(\left(x_{m(q)+k-1}, T\left(\left(x_{m(q)+i-1, m(q)+k-1}\right)_{i=1}^{\infty}\right)\right)\right)\right\} \\
& \quad=\max \left\{d\left(\left(x_{n(q)+k}, x_{m(q)+k}\right), d\left(x_{n(q)+k-1}, x_{n(q)+k}\right), d\left(x_{m(q)+k-1}, x_{m(q)+k}\right)\right\} .\right.
\end{aligned}
$$

Letting $q \rightarrow \infty$ and using (1), (7), (17), and (18), we get

$$
\lim _{q \rightarrow \infty} \beta\left(M_{k}\left(\left(x_{n(q)+i-1, n} \widehat{(q)+k-1}\right)_{i=1}^{\infty},\left(x_{m(q)+i-1, m(\widehat{q)+k}-1}\right)_{i=1}^{\infty}\right)\right)
$$


and

$$
\lim _{q \rightarrow \infty} M_{k}\left(\left(x_{n(q)+i-1, n \widehat{(q)+k-1}}\right)_{i=1}^{\infty},\left(x_{m(q)+i-1, n) \widehat{(q)+k}-1}\right)_{i=1}^{\infty}\right) \geq \varepsilon .
$$

From (17)

$$
\beta\left(M_{k}\left(\left(x_{n(q)+i-1, n(\overline{q)+k-1}}\right)_{i=1}^{\infty},\left(x_{m(q)+i-1, m(q)+k-1}\right)_{i=1}^{\infty}\right)\right) \geq 1 .
$$

Since $\beta \in \mathbb{G}$, we get

$$
\lim _{q \rightarrow \infty} \beta\left(M_{k}\left(\left(x_{n(q)+i-1, n(\overline{q)+k-1}}\right)_{i=1}^{\infty},\left(x_{m(q)+i-1, m(\overline{m)+k-1}}\right)_{i=1}^{\infty}\right)\right)=1 .
$$

Then

$$
\lim _{q \rightarrow \infty} M_{k}\left(\left(x_{n(q)+i-1, n(\widehat{q)+k-1}}\right)_{i=1}^{\infty},\left(x_{m(q)+i-1, m(q)+k-1}\right)_{i=1}^{\infty}\right)=0 .
$$

We get a contradiction to (17). Hence, $\left\{x_{n+k}\right\}_{n \in \mathbb{N}}$ is a Cauchy sequence.

Since $(X, d)$ is complete, there exists $u \in X$ such that

$$
\lim _{n \rightarrow \infty} x_{n+k}=u
$$

We claim that $d\left(\left(T\left((u)_{i=1}^{\infty}\right), u\right)=0\right.$. If we suppose on the contrary that $d\left(\left(u, T\left((u)_{i=1}^{\infty}\right)\right)>0\right.$, then we have

$$
\begin{aligned}
& M\left(\left(x_{n+i-1, \widehat{n+k-1}}\right)_{i=1}^{\infty},\left((u)_{i=1}^{\infty}\right)\right) \\
& \quad=\max \left\{d\left(x_{n+k-1}, u\right), d\left(\left(x_{n+k-1}, T\left(x_{n+i-1, \widehat{n+k-1}}\right)_{i=1}^{\infty}\right), d\left(\left(u, T\left((u)_{i=1}^{\infty}\right)\right)\right\}\right.\right. \\
& \quad=\max \left\{d \left(\left(x_{n+k-1}, u\right), d\left(\left(x_{n+k}, x_{n+k-1}\right), d\left(\left(u, T\left((u)_{i=1}^{\infty}\right)\right)\right\} .\right.\right.\right.
\end{aligned}
$$

Letting $n \rightarrow \infty$ and using (7), (19), we get

$$
\lim _{n \rightarrow \infty} M\left(\left(x_{n+i-1, \widehat{n+k-1}}\right)_{i=1}^{\infty},(u)_{i=1}^{\infty}\right)=d\left(\left(u, T\left((u)_{i=1}^{\infty}\right)\right) \neq 0 .\right.
$$

Then we have

$$
\begin{aligned}
& d\left(\left(u, T\left((u)_{i=1}^{\infty}\right)\right)\right. \\
& \quad \leq d\left(\left(x_{n+k}, u\right)+d\left(\left(x_{n+k}, T\left((u)_{i=1}^{\infty}\right)\right)\right.\right. \\
& \quad=d\left(\left(x_{n+k}, u\right)+d\left(\left(T\left(\left(x_{n+i-1, \widehat{n+k-1}}\right)_{i=1}^{\infty}\right), T\left((u)_{i=1}^{\infty}\right)\right)\right.\right. \\
& \quad \leq d\left(\left(x_{n+k}, u\right)+\beta\left(M\left(\left(x_{n+i-1, \widehat{n+k-1}}\right)_{i=1}^{\infty},(u)_{i=1}^{\infty}\right)\right) M\left(\left(x_{n+i-1, \widehat{n+k-1}}\right)_{i=1}^{\infty},(u)_{i=1}^{\infty}\right)\right. \\
& \quad \leq d\left(\left(x_{n+k}, u\right)+\beta\left(d ( ( u , T ( ( u ) _ { i = 1 } ^ { \infty } ) ) ) d \left(\left(u, T\left((u)_{i=1}^{\infty}\right)\right) .\right.\right.\right.
\end{aligned}
$$

Letting $n \rightarrow \infty$ and using (19), we get $\lim _{n \rightarrow \infty} \beta\left(d\left(\left(u, T\left((u)_{i=1}^{\infty}\right)\right)\right) \geq 1\right.$.

Since $\beta \in \mathbb{G}$, one writes $\lim _{n \rightarrow \infty} \beta\left(d\left(\left(u, T\left((u)_{i=1}^{\infty}\right)\right)\right)=1\right.$. Then we have $\lim _{n \rightarrow \infty} d((u$, $\left.T\left((u)_{i=1}^{\infty}\right)\right)=0$, which implies $d\left(\left(u, T\left((u)_{i=1}^{\infty}\right)\right)=0\right.$. So we get a contradiction. Hence, $d\left(\left(u, T\left((u)_{i=1}^{\infty}\right)\right)=0\right.$, that is, $\left.T\left((u)_{i=1}^{\infty}\right)\right)=u$. 
Remark 2.1 Theorem 2.1 is a proper generalization of Theorem 1.5 since in the case of the simplest operator on $\prod_{i=1}^{\infty} X \rightarrow X$ the contraction condition of Theorem 1.5 is not applicable; on the other hand, the $H_{k}$ contraction (see Definition 2.2) is easily applicable for the infinite case. Also, if we restrict the operator to any finite $k$ dimension through an easy calculation, it is obvious that it is an equivalent statement of Theorem 1.5.

Theorem 2.2 . Let $(X, d)$ be a complete metric space and $T: \prod_{l=1}^{\infty} X \rightarrow X$ be an extended Kannan-Geraghty $H_{k}$ contraction for some $k \in \mathbb{N}$. Then there is $u \in X$ such that $T\left((u)_{i=1}^{\infty}\right)=$ $u$, and for any $x_{1}, \ldots, x_{k} \in X$, the infinite $k$-Picard sequence converges to $u$.

Proof Let $x_{1}, \ldots, x_{k} \in X$. For all $n \in \mathbb{N}$, we define the infinite $k$-Picard sequence as follows:

$$
x_{n+k}:=T\left(\left(x_{n+i-1, \widehat{n+k-1}}\right)_{i=1}^{\infty}\right) \text {. }
$$

We claim that $\lim _{n \rightarrow \infty} d\left(x_{n+k}, x_{n+k+1}\right)=0$. Then we have

$$
\begin{aligned}
& d\left(\left(x_{n+k+1}, x_{n+k+2}\right)\right. \\
& \quad=d\left(\left(T\left(\left(x_{n+i, \widehat{n+k}}\right)_{i=1}^{\infty}\right), T\left(\left(x_{n+i+1, \widehat{n+1+k}}\right)_{i=1}^{\infty}\right)\right)\right. \\
& \quad \leq \frac{\beta\left(d\left(x_{n+k}, x_{n+k+1}\right)\right)}{2}\left(d\left(T\left(\left(x_{n+i, \widehat{n+k}}\right)_{i=1}^{\infty}\right), x_{n+k}\right)+d\left(T\left(\left(x_{n+i+1, \widehat{n+k+1}}\right)_{i=1}^{\infty}\right), x_{n+k+1}\right)\right) \\
& \quad<\frac{1}{2}\left(d\left(x_{n+k}, x_{n+k+1}\right)+d\left(x_{n+k+1}, x_{n+k+2}\right)\right) .
\end{aligned}
$$

Thus, $d\left(x_{n+k+1}, x_{n+k+2}\right)<d\left(x_{n+k}, x_{n+k+1}\right)$. This sequence is decreasing, so there is $r \geq 0$ so that

$$
\lim _{n \rightarrow 0} d\left(x_{n+k}, x_{n+k+1}\right)=r
$$

We claim that $r=0$. If we consider on the contrary and suppose $r>0$, we have

$$
\begin{aligned}
\lim _{n \rightarrow \infty} d\left(x_{n+k+1}, x_{n+k+2}\right) \leq & \frac{\lim _{n \rightarrow \infty} \beta\left(d\left(x_{n+k}, x_{n+k+1}\right)\right)}{2}\left(\lim _{n \rightarrow \infty} d\left(x_{n+k}, x_{n+k+1}\right)\right. \\
& \left.+\lim _{n \rightarrow \infty} d\left(x_{n+k+1}, x_{n+k+2}\right)\right) .
\end{aligned}
$$

Therefore,

$$
\frac{2 \lim _{n \rightarrow \infty} d\left(x_{n+k+1}, x_{n+k+2}\right)}{\lim _{n \rightarrow \infty} d\left(x_{n+k}, x_{n+k+1}\right)+\lim _{n \rightarrow \infty} d\left(x_{n+k+1}, x_{n+k+2}\right)} \leq \lim _{n \rightarrow \infty} \beta\left(d\left(x_{n+k}, x_{n+k+1}\right)\right) .
$$

From (21) we get $\frac{2 r}{2 r} \leq \beta\left(d\left(x_{n+k}, x_{n+k+1}\right)\right)$, and so $\lim _{n \rightarrow \infty} \beta\left(d\left(x_{n+k}, x_{n+k+1}\right)\right) \geq 1$. Since $\beta \in$ $\mathbb{G}$, we have $\lim _{n \rightarrow \infty} \beta\left(d\left(x_{n+k}, x_{n+k+1}\right)\right) \leq 1$. Using the well-known "sandwich theorem", we obtain

$$
\lim _{n \rightarrow \infty} \beta\left(d\left(x_{n+k}, x_{n+k+1}\right)\right)=1 \quad \Longrightarrow \quad \lim _{n \rightarrow \infty} d\left(x_{n+k}, x_{n+k+1}\right)=0 .
$$

Further, we have

$$
d\left(x_{n+k}, x_{m+k}\right)=d\left(T\left(\left(x_{n+i-1, \widehat{n+k-1}}\right)_{i=1}^{\infty}\right), T\left(\left(x_{m+i-1, \widehat{m+k-1}}\right)_{i=1}^{\infty}\right)\right)
$$




$$
\begin{aligned}
\leq & \frac{\beta\left(d\left(x_{n+k-1}, x_{m+k-1}\right)\right)}{2}\left(d\left(T\left(\left(x_{n+i-1, \widehat{n+k-1}}\right)_{i=1}^{\infty}\right), x_{n+k-1}\right)\right) \\
& \left.+d\left(T\left(\left(x_{m+i-1, \widehat{m+k-1}}\right)_{i=1}^{\infty}\right), x_{m+k-1}\right)\right) \\
< & \frac{1}{2}\left(d\left(x_{n+k-1}, x_{n+k}\right)+d\left(x_{m+k-1}, x_{m+k}\right)\right) .
\end{aligned}
$$

For large enough $n, m \in \mathbb{N}$, one has

$$
d\left(x_{n+k}, x_{m+k}\right)<\varepsilon
$$

for fixed $\varepsilon>0$. Then $\left\{x_{n+k}\right\}_{n \in \mathbb{N}}$ is a Cauchy sequence. Since $(X, d)$ is a complete metric space, there exists $u \in X$ such that

$$
\lim _{n \rightarrow \infty} x_{n+k}=u
$$

We claim that $T\left((u)_{i=1}^{\infty}\right)=u$. If we suppose on the contrary that $d\left(u, T\left((u)_{i=1}^{\infty}\right)\right)>0$. Then, by (21) and (23), for arbitrary $\varepsilon>0$ and sufficiently large $n$, we get

$$
\begin{aligned}
d\left(\left(u, T\left((u)_{i=1}^{\infty}\right)\right) \leq\right. & d\left(\left(x_{n+k}, u\right)+d\left(x_{n+k}, T\left((u)_{i=1}^{\infty}\right)\right)\right. \\
= & d\left(\left(x_{n+k}, u\right)+d\left(\left(T\left(\left(x_{n+i-1, \widehat{n+k-1}}\right)_{i=1}^{\infty}\right), T\left((u)_{i=1}^{\infty}\right)\right)\right.\right. \\
\leq & d\left(\left(x_{n+k}, u\right)+\frac{\beta\left(d\left(u, x_{n+k-1}\right)\right)}{2}\left(d\left(T\left(\left(x_{n+i-1, \widehat{n+k-1}}\right)_{i=1}^{\infty}\right), x_{n+k-1}\right)\right.\right. \\
& \left.+d\left(T\left((u)_{i=1}^{\infty}\right), u\right)\right) \\
\leq & \frac{1}{2} d\left(\left(u, T\left((u)_{i=1}^{\infty}\right)\right)+\frac{\varepsilon}{2}+\frac{\varepsilon}{2} \leq \frac{1}{2} d\left(u, T\left((u)_{i=1}^{\infty}\right)+\varepsilon\right.\right. \\
\Longrightarrow & \frac{1}{2} d\left(\left(u, T\left((u)_{i=1}^{\infty}\right)\right) \leq \varepsilon .\right. \text { This is a contradiction. }
\end{aligned}
$$

Hence, $d\left(T\left((u)_{i=1}^{\infty}\right), u\right)=0$, that is, $T\left((u)_{i=1}^{\infty}\right)=u$.

Next, we will provide a new result for a multivalued proper extension of Theorem 1.4, which is also a generalization of Kannan (Theorem 1.3) as a result of Theorem 2.2.

Corollary 2.1 Let $(X, d)$ be a complete metric space and $T$ be a Kannan-Geraghty $H_{k}$ contraction. Then $T$ has a fixed point and every $k$-Picard sequence for $T$ converges to $u$.

Proof Let us choose $x_{0}, \ldots, x_{k} \in X$. We define a $k$-Picard sequence by

$$
x_{n+k}:=T\left(x_{n}, \ldots, x_{n+k-1}\right)
$$

If we follow the same steps as in the proof of Theorem 2.2, we get the required result.

Remark 2.2 For $k=1$, we get Theorem 1.4 in [3] which proves that Corollary 2.1 is a proper generalization of Theorem 1.4. 
Theorem 2.3 Let $(X, d)$ be a complete metric space and $T: \prod_{l=1}^{\infty} X \rightarrow X$ be an extended Fisher-Geraghty $H_{k}$ contraction for some $k \in \mathbb{N}$. Then there exists $u \in X$ such that $T\left((u)_{i=1}^{\infty}\right)=u$, and for any $x_{1}, x_{2}, \ldots x_{k}$, the infinite $k$-Picard sequence converges to $u$.

Proof Let $x_{1}, \ldots, x_{k} \in X$. For all $n \in \mathbb{N}$, we define an infinite $k$ - Picard sequence by

$$
x_{n+k}:=T\left(\left(x_{n+i-1, \widehat{n+k-1}}\right)_{i=1}^{\infty}\right) \text {. }
$$

We claim $\lim _{n \rightarrow \infty} d\left(x_{n+k}, d_{n+k+1}\right)=0$.

Then we have

$$
\begin{aligned}
d\left(\left(x_{n+k+1}, x_{n+k+2}\right)=\right. & d\left(\left(T\left(\left(x_{n+i, \widehat{n+k}}\right)_{i=1}^{\infty}\right), T\left(\left(x_{n+i+1, \widehat{n+1+k}}\right)_{i=1}^{\infty}\right)\right)\right. \\
\leq & \frac{\beta\left(d\left(x_{n+k}, x_{n+k+1}\right)\right)}{2}\left(d\left(T\left(\left(x_{n+i, \widehat{n+k}}\right)_{i=1}^{\infty}\right), x_{n+k+1}\right)\right. \\
& \left.+d\left(T\left(\left(x_{n+i, \widehat{n+k+1}}\right)_{i=1}^{\infty}\right), x_{n+k}\right)\right) \\
< & \frac{1}{2}\left(d\left(x_{n+k+1}, x_{n+k+1}\right)+d\left(x_{n+k}, x_{n+k+2}\right)\right) \\
< & \frac{1}{2}\left(d\left(x_{n+k}, x_{n+k+1}\right)+d\left(x_{n+k+1}, x_{n+k+2}\right)\right) .
\end{aligned}
$$

We have $d\left(x_{n+k+1}, x_{n+k+2}\right)<d\left(x_{n+k}, x_{n+k+1}\right)$. This sequence is decreasing, so there is $r \geq 0$ such that

$$
\lim _{n \rightarrow 0} d\left(x_{n+k}, x_{n+k+1}\right)=r
$$

We claim that $r=0$. If we suppose on the contrary that $r>0$. One has

$$
\begin{aligned}
& \lim _{n \rightarrow \infty} d\left(x_{n+k+1}, x_{n+k+2}\right) \\
& \quad \leq \frac{\lim _{n \rightarrow \infty} \beta\left(d\left(x_{n+k}, x_{n+k+1}\right)\right)}{2}\left(\lim _{n \rightarrow \infty} d\left(x_{n+k}, x_{n+k+1}\right)+\lim _{n \rightarrow \infty} d\left(x_{n+k+1}, x_{n+k+2}\right)\right) .
\end{aligned}
$$

Thus,

$$
\frac{2 \lim _{n \rightarrow \infty} d\left(x_{n+k+1}, x_{n+k+2}\right)}{\lim _{n \rightarrow \infty} d\left(x_{n+k}, x_{n+k+1}\right)+\lim _{n \rightarrow \infty} d\left(x_{n+k+1}, x_{n+k+2}\right)} \leq \lim _{n \rightarrow \infty} \beta\left(d\left(x_{n+k}, x_{n+k+1}\right)\right) .
$$

Using (26), we obtain $\frac{2 r}{2 r} \leq \lim _{n \rightarrow \infty} \beta\left(d\left(x_{n+k}, x_{n+k+1}\right)\right)$. Hence,

$$
\lim _{n \rightarrow \infty} \beta\left(d\left(x_{n+k}, x_{n+k+1}\right)\right) \geq 1 .
$$

Since $\beta \in \mathbb{G}$, we have $\lim _{n \rightarrow \infty} \beta\left(d\left(x_{n+k}, x_{n+k+1}\right)\right) \leq 1$.

At the limit, we have

$$
\lim _{n \rightarrow \infty} \beta\left(d\left(x_{n+k}, x_{n+k+1}\right)\right)=1
$$

Then $\lim _{n \rightarrow \infty} d\left(x_{n+k}, x_{n+k+1}\right)=0$. We claim that $\left\{x_{n+k}\right\}_{n \in \mathbb{N}}$ is Cauchy, and we want to prove this by contradiction. Then, using the contrary, there exists $\varepsilon>0$ such that we can find 
subsequences $\left\{x_{m(q)+k}\right\}_{p \in \mathbb{N}},\left\{x_{n(q)+k}\right\}_{p \in \mathbb{N}}$ with $m(q)>n(q)>q$ such that, for every $q$, we have

$$
d\left(\left(x_{m(q)+k}, x_{n(q)+k}\right) \geq \varepsilon .\right.
$$

Moreover, corresponding to each $n(q)$ we can choose $m(q)$ satisfying (28) so that

$$
d\left(\left(x_{m(q)+k-1}, x_{n(q)+k}\right)<\varepsilon .\right.
$$

Using (28), (29) and the triangle inequality, we get

$$
\begin{aligned}
d\left(\left(x_{m(q)+k-1}, x_{n(q)+k-1}\right)\right. & \leq d\left(\left(x_{m(q)+k-1}, x_{n(q)+k}\right)+d\left(\left(x_{n(q)+k-1}, x_{n(q)+k}\right)\right.\right. \\
& <\varepsilon+\xi\left(\left(x_{n(q)+k-1}, x_{n(q)+k}\right)\right.
\end{aligned}
$$

and

$$
\begin{aligned}
\varepsilon & \leq d\left(\left(x_{n(q)+k}, x_{m(q)+k}\right)\right. \\
& \leq d\left(\left(x_{n(q)+k}, x_{n(q)+k-1}\right)+d\left(\left(x_{n(q)+k-1}, x_{m(q)+k-1}\right)+d\left(\left(x_{m(q)+k-1}, x_{m(q)+k}\right) .\right.\right.\right.
\end{aligned}
$$

Letting $q \rightarrow \infty$ in (30) and (27), we get

$$
\begin{aligned}
& \lim _{q \rightarrow \infty} d\left(\left(x_{m(q)+k-1}, x_{n(q)+k-1}\right)=\varepsilon,\right. \\
& d\left(x_{n(q)+k}, x_{m(q)+k}\right) \\
&=d\left(T\left(\left(x_{n(q)+i-1, n(\bar{q})+k-1}\right)_{i=1}^{\infty}\right), T\left(\left(x_{m(q)+i-1, m(q)+k-1}\right)_{i=1}^{\infty}\right)\right) \\
& \leq \frac{\beta\left(d\left(x_{n(q)+k-1}, x_{m(q)+k-1}\right)\right)}{2}\left(d\left(T\left(\left(x_{n(q)+i-1, n(\bar{q})+k-1}\right)_{i=1}^{\infty}\right), x_{m(q)+k-1}\right)\right. \\
&\left.\quad+d\left(T\left(\left(x_{m(q)+i-1, m(q)+k-1}\right)_{i=1}^{\infty}\right), x_{n(q)+k-1}\right)\right) \\
&<\frac{1}{2}\left(d\left(x_{n(q)+k}, x_{m(q)+k-1}\right)+d\left(x_{m(q)+k}, x_{n(q)+k-1}\right)\right) \\
& \leq \frac{1}{2}\left(\varepsilon+d\left(x_{m(q)+k}, x_{m(q)+k-1}\right)+d\left(x_{m(q)+k-1}, x_{n(q)+k}\right)+d\left(x_{n(q)+k}, x_{n(q)+k-1}\right)\right) \\
& \leq \frac{1}{2}\left(2 \varepsilon+d\left(x_{m(q)+k}, x_{m(q)+k-1}\right)+d\left(x_{n(q)+k}, x_{n(q)+k-1}\right)\right) .
\end{aligned}
$$

Then from (31) and (27) we get a contradiction. Then

$$
\left\{x_{n+k}\right\}_{n \in \mathbb{N}} \text { is a Cauchy sequence. }
$$

Since $(X, d)$ is complete, there exists $u \in X$ such that

$$
\lim _{n \rightarrow \infty} x_{n+k}=u
$$

We claim that $T\left((u)_{i=1}^{\infty}\right)=u$. If we suppose on the contrary that $d\left(u, T\left((u)_{i=1}^{\infty}\right)\right)>0$, then, by (26) and (33), for arbitrary $\varepsilon>0$ and sufficiently large $n$, we get

$$
d\left(\left(u, T\left((u)_{i=1}^{\infty}\right)\right)\right.
$$




$$
\begin{aligned}
\leq & d\left(\left(x_{n+k}, u\right)+d\left(x_{n+k}, T\left((u)_{i=1}^{\infty}\right)\right)\right. \\
= & d\left(\left(x_{n+k}, u\right)+d\left(\left(T\left(\left(x_{n+i-1, \widehat{n+k-1}}\right)_{i=1}^{\infty}\right), T\left((u)_{i=1}^{\infty}\right)\right)\right.\right. \\
\leq & d\left(\left(x_{n+k}, u\right)+\frac{\beta\left(\xi\left(u, x_{n+k-1}\right)\right)}{2}\left(d\left(T\left(\left(x_{n+i-1, \widehat{n+k-1}}\right)_{i=1}\right)^{\infty}\right), u\right)+d\left(T\left((u)_{i=1}^{\infty}\right), x_{n+k-1}\right)\right) \\
\leq & d\left(\left(x_{n+k}, u\right)+\frac{\beta\left(d\left(u, x_{n+k-1}\right)\right)}{2}\left(d\left(T\left(\left(x_{n+i-1, \widehat{n+k-1}}\right)_{i=1}\right)^{\infty}\right), u\right)\right. \\
& \left.+d\left(T\left((u)_{i=1}^{\infty}\right), u\right)+d\left(u, x_{n+k-1}\right)\right) \\
\leq & \frac{1}{2} d\left(\left(u, T\left((u)_{i=1}^{\infty}\right)\right)+\frac{\varepsilon}{3}+\frac{\varepsilon}{6}\right. \\
\leq & \frac{1}{2} d\left(u, T\left((u)_{i=1}^{\infty}\right)+\frac{1}{2} \varepsilon .\right.
\end{aligned}
$$

That is, $d\left(\left(u, T\left((u)_{i=1}^{\infty}\right)\right) \leq \varepsilon\right.$ for any arbitrary $\varepsilon>0$. Hence, $d\left(T\left((u)_{i=1}^{\infty}\right), u\right)=0$, which implies $T\left((u)_{i=1}^{\infty}\right)=u$.

Corollary 2.2 Let $(X, d)$ be a complete metric space and $T$ be a Fisher-Geraghty $H_{k}$ contraction. Then $T$ has a fixed point.

Proof Choose any $x_{0}, \ldots, x_{k} \in X$. Define

$$
x_{n+k}:=T\left(x_{n}, \ldots, x_{n+k-1}\right) .
$$

Using the same steps as in the proof of Theorem 2.3, we get the conclusion.

Remark 2.3 For $k=1$, we get a new type of extension of Theorem 1.3 proved in [2], and for any $k$, Corollary 2.2 also gives the multidimensional extension of the same theorem stated in [2].

Remark 2.4 The Banach fixed point theorem [4], Theorem 1.1, Theorem 1.2, Theorem 1.3, Theorem 1.4, and Theorem 1.5 are all applicable only in complete metric spaces as well as the theorems described in [1], but Theorem 2.1, Theorem 2.2, Theorem 2.3, which we have proved, are talking about the space ${ }_{i=1}^{\infty} X$ (where $X$ is a complete metric space) which is indeed metrizable, but might not be complete.

\section{Applications to integral equations}

In [41], Singh et al. introduced the notion of multi-dimensional Fredholm integral equations by taking into account $n \in \mathbb{N}$ as the dimension of the equation

$$
\begin{aligned}
u\left(x_{1}, \ldots, x_{n}\right)= & f\left(x_{1}, \ldots, x_{n}\right) \\
& +\underbrace{\int_{0}^{1} \int_{0}^{1} \ldots \int_{0}^{1} K\left(x_{1}, \ldots, x_{n}, y_{1}, \ldots, y_{n}\right) u\left(y_{1}, \ldots, y_{n}\right) d y_{1} \ldots d y_{n},}_{\text {n-times }}
\end{aligned}
$$

where $\left(x_{1}, \ldots, x_{n}\right) \in D=\underbrace{([0,1] \times[0,1] \times \cdots \times[0,1])}_{\mathrm{n} \text {-times }}$ and $f\left(x_{1}, \ldots, x_{n}\right), K\left(x_{1}, \ldots, x_{n}, y_{1}\right.$, $\left.\ldots, y_{n}\right)$ are known continuous functions defined on $D$ and $D^{2}$, respectively, where $u\left(x_{1}\right.$, $\left.\ldots, x_{n}\right)$ is an unknown function. 
The Fredholm integral equations play an important role in modeling of physics phenomena described by two or three dimensions. In the same way, they have applications in astrophysics models thinking of the four dimensions of a neutron star or a black hole.

Thinking of these aspects, if we extend to infinity the dimension " $\mathrm{n}$ " of the previous multi-dimensional integral equation, we introduce a new notion, the infinite dimensional Fredholm integral equation, as follows:

$$
\begin{aligned}
u\left(\left(t_{i, \widehat{k}}\right)_{i=1}^{\infty}\right)= & f\left(\left(t_{i, \widehat{k}}\right)_{i=1}^{\infty}\right) \\
& +\int_{0}^{1} \ldots \int_{0}^{1} \ldots K\left(\left(t_{i, \widehat{k}}\right)_{i=1}^{\infty},\left(s_{i, \hat{k}}\right)_{i=1}^{\infty}\right) u\left(\left(s_{i, \widehat{k}}\right)_{i=1}^{\infty}\right) d s_{1} \cdots d s_{n} \cdots,
\end{aligned}
$$

where $f\left(\left(t_{i, \widehat{k}}\right)_{i=1}^{\infty}\right):[0,1] \rightarrow \mathbb{R}$ and $K:[0,1] \times \mathbb{R} \rightarrow \mathbb{R}$ are two known continuous functions and $u\left(\left(t_{i, \widehat{k}}\right)_{i=1}^{\infty}\right)$ is an unknown function.

Further, let us give our first application of the main results of this paper by proving the existence of a solution of infinite dimensional Fredholm integral equation (35).

Theorem 3.1 Let $X=C([0,1], \mathbb{R})$ be the set of real continuous functions on $[0,1]$, and let $d: X \times X \rightarrow \mathbb{R}_{+}$be given by

$$
d\left(\left(\left(u_{i, \widehat{k}}\right)_{i=1}^{\infty}, T\left(u_{i, \widehat{k}}\right)_{i=1}^{\infty}\right)=\sup _{t \in[0,1]}\left|\left(u_{i, \widehat{k}}\right)_{i=1}^{\infty}-T\left(u_{i, \hat{k}}\right)_{i=1}^{\infty}\right|\right.
$$

Define $T: \prod_{i=1}^{\infty} X \rightarrow X$ by

$$
\begin{aligned}
\operatorname{Tu}\left(\left(t_{i, \widehat{k}}\right)_{i=1}^{\infty}\right)= & f\left(\left(t_{i, \widehat{k}}\right)_{i=1}^{\infty}\right) \\
& +\int_{0}^{1} \ldots \int_{0}^{1} \ldots K\left(\left(t_{i, \widehat{k}}\right)_{i=1}^{\infty},\left(s_{i, \widehat{k}}\right)_{i=1}^{\infty}\right) u\left(\left(s_{i, \widehat{k}}\right)_{i=1}^{\infty}\right) d s_{1} \cdots d s_{n} \cdots
\end{aligned}
$$

Assume that the following hold:

(i) $\left|u\left(\left(t_{i, \widehat{k}}\right)_{i=1}^{\infty}\right)-T u\left(\left(t_{i, \widehat{k}}\right)_{i=1}^{\infty}\right)\right| \leq \frac{1}{2}\left(\left|T\left(\left(u_{i, \widehat{k}}\right)_{i=1}^{\infty}\right)-u_{k}\right|+\left|T\left(\left(u_{i+1, \widehat{k+1}}\right)_{i=1}^{\infty}\right)-u_{k+1}\right|\right)$;

(ii) There exists a constant $\delta \in(0,1)$ so that $K\left(\left(t_{i, \hat{k}}\right)_{i=1}^{\infty},\left(s_{i, \widehat{k}}\right)_{i=1}^{\infty}\right)<\delta$;

(iii) Let $\beta:[0, \infty) \rightarrow[0,1)$ be a lower bounded function and $\gamma>1$ be large enough such that $\frac{1}{\gamma}<\beta(z)$ for every $z \in X$.

Then the infinite dimensional Fredholm integral equation (35) has a solution.

Proof We can prove the existence of a solution of infinite dimensional Fredholm integral equation if we show that the operator $T$ defined by (37) has a fixed point.

We can easy remark that the space $X=(C[0,1], \mathbb{R})$ endowed with the metric $d$ defined by relation (36) forms a complete metric space. Then we shall show that all the hypotheses of Theorem 2.2 are verified.

We have the following estimation:

$$
\begin{aligned}
& \left|\operatorname{Tu}\left(\left(t_{i, \widehat{k}}\right)_{i=1}^{\infty}\right)-T^{2} u\left(\left(t_{i, \widehat{k}}\right)_{i=1}^{\infty}\right)\right| \\
& =\mid \int_{0}^{1} \ldots \int_{0}^{1} \ldots K\left(\left(t_{i, \hat{k}}\right)_{i=1}^{\infty},\left(s_{i, \widehat{k}}\right)_{i=1}^{\infty}\right) u\left(\left(s_{i, \widehat{k}}\right)_{i=1}^{\infty}\right) d s_{1} \ldots d s_{n} \ldots \\
& \quad-\int_{0}^{1} \ldots \int_{0}^{1} \ldots K\left(\left(t_{i, \widehat{k}}\right)_{i=1}^{\infty},\left(s_{i, \widehat{k}}\right)_{i=1}^{\infty}\right) T u\left(\left(s_{i, \widehat{k}}\right)_{i=1}^{\infty}\right) d s_{1} \ldots d s_{n} \ldots \mid
\end{aligned}
$$




$$
\begin{aligned}
& \leq \int_{0}^{1} \ldots \int_{0}^{1} \ldots K\left(\left(t_{i, \widehat{k}}\right)_{i=1}^{\infty},\left(s_{i, \widehat{k}}\right)_{i=1}^{\infty}\right)\left|u\left(\left(s_{i, \widehat{k}}\right)_{i=1}^{\infty}\right)-T u\left(\left(s_{i, \widehat{k}}\right)_{i=1}^{\infty}\right)\right| d s_{1} \ldots d s_{n} \ldots \\
& \leq \frac{\delta}{2 \gamma}\left(\left|T\left(\left(u_{i, \widehat{k}}\right)_{i=1}^{\infty}\right)-u_{k}\right|+\left|T\left(\left(u_{i+1, \widehat{k+1}}\right)_{i=1}^{\infty}\right)-u_{k+1}\right|\right) \int_{0}^{1} \ldots \int_{0}^{1} \ldots d s_{1} \ldots d s_{n} \ldots \\
& =\frac{\delta}{2 \gamma}\left(\left|T\left(\left(u_{i, \widehat{k}}\right)_{i=1}^{\infty}\right)-u_{k}\right|+\left|T\left(\left(u_{i+1, \widehat{k+1}}\right)_{i=1}^{\infty}\right)-u_{k+1}\right|\right) .
\end{aligned}
$$

Taking supremum on both sides, we get

$$
\begin{aligned}
& \sup _{t \in[0,1]}\left|\operatorname{Tu}\left(\left(t_{i, \widehat{k}}\right)_{i=1}^{\infty}\right)-T^{2} u\left(\left(t_{i, \widehat{k}}\right)_{i=1}^{\infty}\right)\right| \\
& \quad \leq \frac{\delta}{2 \gamma} \sup _{t \in[0,1]}\left(\left|T\left(\left(u_{i, \widehat{k}}\right)_{i=1}^{\infty}\right)-u_{k}\right|+\left|T\left(\left(u_{i+1, \widehat{k+1}}\right)_{i=1}^{\infty}\right)-u_{k+1}\right|\right) .
\end{aligned}
$$

Further, since $\frac{1}{\gamma}<\beta(z)$, for $z=d\left(u_{k}, u_{k+1}\right)$ and $\delta \in(0,1)$, we obtain

$$
\begin{aligned}
& d\left(\left(\operatorname{Tu}\left(\left(t_{i, \widehat{k}}\right)_{i=1}^{\infty}\right), T^{2} u\left(\left(t_{i, \widehat{k}}\right)_{i=1}^{\infty}\right)\right)\right. \\
& \quad \leq \frac{\delta \beta\left(d\left(u_{k}, u_{k+1}\right)\right)}{2}\left(d \left(\left(T\left(\left(u_{i, \widehat{k}}\right)_{i=1}^{\infty}\right), u_{k}\right)+d\left(\left(T\left(\left(u_{i+1, \widehat{k+1}}\right)_{i=1}^{\infty}\right), u_{k+1}\right)\right)\right.\right. \\
& \quad \leq \frac{\beta\left(d\left(u_{k}, u_{k+1}\right)\right)}{2}\left(d \left(\left(T\left(\left(u_{i, \widehat{k}}\right)_{i=1}^{\infty}\right), u_{k}\right)+d\left(\left(T\left(\left(u_{i+1, \widehat{k+1}}\right)_{i=1}^{\infty}\right), u_{k+1}\right)\right) .\right.\right.
\end{aligned}
$$

In conclusion, all the hypotheses of Theorem 2.2 are accomplished. Then the operator $T$ has a fixed point, which means the infinite dimensional Fredholm integral equation (35) has a solution.

The following application involves another type of integral equations: Urysohn type integral equations. We extend the known cases of this type of integral equations to infinite dimensional Urysohn integral equations:

$$
\begin{aligned}
u\left(\left(t_{i, \widehat{k}}\right)_{i=1}^{\infty}\right)= & f\left(\left(t_{i, \widehat{k}}\right)_{i=1}^{\infty}\right) \\
& +\int_{a}^{b} \ldots \int_{a}^{b} \ldots P\left(\left(t_{i, \widehat{k}}\right)_{i=1}^{\infty},\left(s_{i, \widehat{k}}\right)_{i=1}^{\infty}, u\left(\left(s_{i, \widehat{k}}\right)_{i=1}^{\infty}\right)\right) d s_{1} \cdots d s_{n} \cdots,
\end{aligned}
$$

where $f\left(\left(t_{i, \widehat{k}}\right)_{i=1}^{\infty}\right):[a, b] \rightarrow \mathbb{R}$ and $P:[a, b] \times[a, b] \times \mathbb{R} \rightarrow \mathbb{R}$ are two known continuous functions and $u\left(\left(t_{i, \widehat{k}}\right)_{i=1}^{\infty}\right)$ is an unknown function.

For this new type of Urysohn integral equation, let us give the following result.

Theorem 3.2 Let $X=C([a, b], \mathbb{R})$ be the set of real continuous functions on $[a, b]$ endowed with the Bielecki norm $d: X \times X \rightarrow \mathbb{R}_{+}$given by

$$
d\left(\left(\left(u_{i, \widehat{k}}\right)_{i=1}^{\infty}, T\left(u_{i, \widehat{k}}\right)_{i=1}^{\infty}\right)=\left\|\left(u_{i, \widehat{k}}\right)_{i=1}^{\infty}\right\|_{B}=\sup _{t \in[a, b]}\left|u\left(t_{i, \widehat{k}}\right)_{i=1}^{\infty}\right| \cdot e^{-\tau t}, \quad \text { with } \tau>1 .\right.
$$

Define $T: \prod_{i=1}^{\infty} X \rightarrow X$ by

$$
\begin{aligned}
\operatorname{Tu}\left(\left(t_{i, \widehat{k}}\right)_{i=1}^{\infty}\right)= & f\left(\left(t_{i, \widehat{k}}\right)_{i=1}^{\infty}\right) \\
& +\int_{a}^{b} \ldots \int_{a}^{b} \ldots P\left(\left(t_{i, \widehat{k}}\right)_{i=1}^{\infty},\left(s_{i, \widehat{k}}\right)_{i=1}^{\infty}, u\left(\left(s_{i, \widehat{k}}\right)_{i=1}^{\infty}\right)\right) d s_{1} \cdots d s_{n} \cdots
\end{aligned}
$$


Assume that there is $\varpi>0$ so that

$$
\begin{aligned}
& \left|P\left(\left(t_{i, \widehat{k}}\right)_{i=1}^{\infty},\left(s_{i, \hat{k}}\right)_{i=1}^{\infty}, u\left(\left(s_{i, \widehat{k}}\right)_{i=1}^{\infty}\right)\right)-P\left(\left(t_{i, \widehat{k}}\right)_{i=1}^{\infty},\left(s_{i, \widehat{k}}\right)_{i=1}^{\infty}, T u\left(\left(s_{i, \widehat{k}}\right)_{i=1}^{\infty}\right)\right)\right| \\
& \quad \leq \frac{1}{\varpi}\left|\left(u_{i, \widehat{k}}\right)_{i=1}^{\infty}-T\left(u_{i, \widehat{k}}\right)_{i=1}^{\infty}\right| .
\end{aligned}
$$

Let $\beta(z):[0, \infty) \rightarrow[0,1)$ be a lower bounded function such that, for $\varpi>1$ large enough, $\frac{1}{\omega}<\beta(z)$ for $z \in X$.

Then the infinite dimensional Urysohn integral equation (38) has a solution.

Proof It is easy to check that the space $X=(C[0,1], \mathbb{R})$ endowed with the metric $d$ defined by relation (39) is a complete metric space.

To prove the existence of a solution of infinite dimensional Urysohn integral equation, we shall show that the operator $T$ defined by (40) has a fixed point.

We have the following estimation:

$$
\begin{aligned}
&\left|T u\left(\left(t_{i, \hat{k}}\right)_{i=1}^{\infty}\right)-T^{2} u\left(\left(t_{i, \widehat{k}}\right)_{i=1}^{\infty}\right)\right| \\
&=\mid \int_{a}^{b} \ldots \int_{a}^{b} \ldots P\left(\left(t_{i, \widehat{k}}\right)_{i=1}^{\infty},\left(s_{i, \hat{k}}\right)_{i=1}^{\infty}, u\left(\left(s_{i, \widehat{k}}\right)_{i=1}^{\infty}\right)\right) d s_{1} \ldots d s_{n} \ldots \\
& \quad-\int_{a}^{b} \ldots \int_{a}^{b} \ldots P\left(\left(t_{i, \hat{k}}\right)_{i=1}^{\infty},\left(s_{i, \widehat{k}}\right)_{i=1}^{\infty}, T u\left(\left(s_{i, \widehat{k}}\right)_{i=1}^{\infty}\right)\right) d s_{1} \ldots d s_{n} \ldots \mid \\
& \leq \int_{a}^{b} \ldots \int_{a}^{b} \ldots \mid P\left(\left(t_{i, \hat{k}}\right)_{i=1}^{\infty},\left(s_{i, \widehat{k}}\right)_{i=1}^{\infty}, u\left(\left(s_{i, \widehat{k}}\right)_{i=1}^{\infty}\right)\right) \\
& \quad-P\left(\left(t_{i, \widehat{k}}^{\infty}\right)_{i=1}^{\infty},\left(s_{i, \widehat{k}}\right)_{i=1}^{\infty}, T u\left(\left(s_{i, \widehat{k}}\right)_{i=1}^{\infty}\right)\right) \mid d s_{1} \ldots d s_{n} \ldots \\
& \leq \frac{1}{\varpi} \int_{a}^{b} \ldots \int_{a}^{b} \ldots\left|\left(u_{i, \widehat{k}}\right)_{i=1}^{\infty}-T\left(u_{i, \widehat{k}}\right)_{i=1}^{\infty}\right| d s_{1} \ldots d s_{n} \ldots \\
& \leq \frac{e^{\tau t}}{\varpi}\left|\left(u_{i, \widehat{k}}\right)_{i=1}^{\infty}-T\left(u_{i, \hat{k}}\right)_{i=1}^{\infty}\right| e^{-\tau t} \int_{a}^{b} \ldots \int_{a}^{b} \ldots d s_{1} \ldots d s_{n} \ldots \\
& \leq \frac{e^{\tau t}}{\varpi}\left|\left(u_{i, \widehat{k}}\right)_{i=1}^{\infty}-T\left(u_{i, \hat{k}}\right)_{i=1}^{\infty}\right| e^{-\tau t} .
\end{aligned}
$$

Applying maximum on both sides, we get

$$
\max _{t \in[a, b]}\left|\operatorname{Tu}\left(\left(t_{i, \hat{k}}\right)_{i=1}^{\infty}\right)-T^{2} u\left(\left(t_{i, \hat{k}}\right)_{i=1}^{\infty}\right)\right| e^{-\tau t} \leq \frac{1}{\varpi} \max _{t \in[a, b]}\left|\left(u_{i, \widehat{k}}\right)_{i=1}^{\infty}-T\left(u_{i, \widehat{k}}\right)_{i=1}^{\infty}\right| e^{-\tau t} .
$$

Then we have

$$
d\left(\left(T u\left(\left(t_{i, \hat{k}}\right)_{i=1}^{\infty}\right), T^{2} u\left(\left(t_{i, \widehat{k}}\right)_{i=1}^{\infty}\right)\right)\right) \leq \frac{1}{\varpi} d\left(\left(\left(u_{i, \widehat{k}}\right)_{i=1}^{\infty}, T\left(u_{i, \widehat{k}}\right)_{i=1}^{\infty}\right) .\right.
$$

Since $\frac{1}{\omega}<\beta(z)$ and for $z=M_{k}\left(\left(u_{i, \hat{k}}\right)_{i=1}^{\infty},\left(u_{i+1, \widehat{k+1}}\right)_{i=1}^{\infty}\right)$, we obtain

$$
\begin{aligned}
& d\left(\left(\operatorname{Tu}\left(\left(t_{i, \widehat{k}}\right)_{i=1}^{\infty}\right), T^{2} u\left(\left(t_{i, \widehat{k}}\right)_{i=1}^{\infty}\right)\right)\right. \\
& \quad \leq \beta\left(M_{k}\left(\left(u_{i, \widehat{k}}\right)_{i=1}^{\infty},\left(u_{i+1, \widehat{k+1}}\right)_{i=1}^{\infty}\right)\right) d\left(\left(\left(u_{i, \widehat{k}}\right)_{i=1}^{\infty}, T\left(u_{i, \widehat{k}}\right)_{i=1}^{\infty}\right)\right. \\
& \quad \leq \beta\left(M_{k}\left(\left(u_{i, \widehat{k}}^{\infty}\right)_{i=1}^{\infty},\left(u_{i+1, \widehat{k+1}}\right)_{i=1}^{\infty}\right)\right) M_{k}\left(\left(u_{i, \widehat{k}}\right)_{i=1}^{\infty},\left(u_{i+1, \widehat{k+1}}\right)_{i=1}^{\infty}\right) .
\end{aligned}
$$


Then all the conditions of Theorem 2.1 are satisfied, so the operator $T$ has a fixed point. Then the infinite dimensional Urysohn type integral equation (38) has a solution.

\section{Conclusion}

Here, in this article we have shown some metric combinatorial arguments that can be profitably applied to extend Geraghty's theorem, Kannan-Geraghty theorem, and Fisher's theorem as well as a particular extension for the infinite cases. This idea could also motivate to find a similar infinite extension of the other contractions and their related operators, to find fixed points or coincidence points. We also defined two new types of infinite integral equations which would encourage the study of the infinite integral equations and their solutions. From the discussion we have some interesting problems as follows:

1. Can a similar concept be extended to the notion of coincidence points?

2. We have discussed the fixed point theorem for the contraction from countable products of the complete metric space $X$ (with respect to uniform metric topology) to the space $X$. Can it further be extended to an uncountable product of a complete metric space which is not necessarily a metric space?

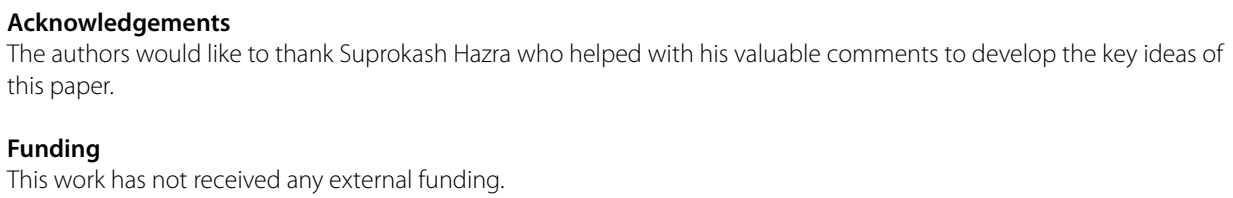

\section{Publisher's Note}

Springer Nature remains neutral with regard to jurisdictional claims in published maps and institutional affiliations.

Received: 28 July 2021 Accepted: 7 September 2021 Published online: 16 October 2021

\section{References}

1. Shumrani, M.Al., Aydi, H., Hazra, S., Ozel, C.: Geraghty extension to K-dimension. SER Math. Inform. 33(2), 197-202 (2018)

2. Fisher, B.: A fixed point theorem. Math. Mag. 48(4), 223-225 (1975)

3. Fogh, F., Behnamian, S., Pashaie, F.: On Kannan-Geraghty as an extension of Kannan maps. Int. J. Maps in Mathematics 2(41), 1-13 (2019)

4. Banach, S.: Sur les opérations dans les ensembles abstraits et leur application aux équations intégrales. Fundam. Math. 3, 133-181 (1922)

5. Javed, K., Uddin, F., Aydi, H., Mukheimer, A., Arshad, M.: Ordered-theoretic fixed point results in fuzzy b-metric spaces with an application. J. Math. 2021, Article ID 6663707 (2021)

6. Asadi, M., Gabeleh, M., Vetro, C.: A new approach to the generalization of Darbo's fixed point problem by using simulation functions with application to integral equations. Results Math. 78, 86 (2019)

7. Mohammed, P.O., Aydi, H., Kashuri, A., Hamed, Y.S., Abualnaja, K.M.: Midpoint inequalities in fractional calculus defined using positive weighted symmetry function kernels. Symmetry 13, 550 (2021) 
8. Alqahtani, B., Aydi, H., Karapinar, E., Rakocević, V.: A solution for Volterra fractional integral equations by hybrid contractions. Mathematics 7(8), 694 (2019)

9. Karapinar, E., Fulga, A., Rashid, M., Shahid, L., Aydi, H.: Large contractions on quasi-metric spaces with an application to nonlinear fractional differential equations. Mathematics 7(5), 444 (2019)

10. Gabeleh, M., Asadi, M., Karapınar, E.: Best proximity results on condensing operators via measure of noncompactness with application to integral equations. Thai J. Math. 18(3), 1519-1535 (2020)

11. Ali, M.U., Aydi, H., Alansari, M.: New generalizations of set valued interpolative Hardy-Rogers type contractions in b-metric spaces. J. Funct. Spaces 2021, Article ID 6641342 (2021)

12. Javed, K., Aydi, H., Uddin, F., Arshad, M.: On orthogonal partial b-metric spaces with an application. J. Math. 2021, Article ID $6692063(2021)$

13. Nikbakhtsarvestani, F., Vaezpour, S.M., Asadi, M.: $F(\psi, \varphi)$-contraction in terms of measure of noncompactness with application for nonlinear integral equations. J. Inequal. Appl. 2017(1), 271 (2017)

14. Marasi, H.R., Aydi, H.: Existence and uniqueness results for two-term nonlinear fractional differential equations via a fixed point technique. J. Math. 2021, Article ID 6670176 (2021)

15. Hammad, H.A., Aydi, H., Mlaiki, N.: Contributions of the fixed point technique to solve the 2D Volterra integral equations, Riemann — Liouville fractional integrals, and Atangana—Baleanu integral operators. Adv. Differ. Equ. 2021, 97 (2021)

16. Singh, D., Chauhan, V., Asadi, M.: Some remarks on tripled fixed point theorems for a sequence of mappings satisfying Geraghty contraction with applications. Commun. Nonlinear Anal. 3, 68-86 (2017)

17. Mlaiki, N., Souayah, N., Abdeljawad, T., Aydi, H.: A new extension to the controlled metric type spaces endowed with a graph. Adv. Differ. Equ. 2021, 94 (2021)

18. Hammad, H.A., Aydi, H., Gaba, Y.U.: Exciting fixed point results on a novel space with supportive applications. J. Funct. Spaces 2021, Article ID 6613774 (2021)

19. Aydi, H., Jeli, M., Samet, B.: On positive solutions for a fractional thermostat model with a convex-concave source term via $\psi$-Caputo fractional derivative. Mediterr. J. Math. 17(1), 16 (2020)

20. Parvaneh, V., Haddadi, M.R., Aydi, H.: On best proximity point results for some type of mappings. J. Funct. Spaces 2020, Article ID 6298138 (2020)

21. Karapinar, E., Agarwal, R.P., Aydi, H.: Interpolative Reich-Rus-Ciric type contractions on partial metric spaces. Mathematics 6, 256 (2018)

22. Karapinar, E., Shahi, P., Tas, K.: Generalized $\alpha$ - $\psi$-contractive type mappings of integral type and related fixed point theorems. J. Inequal. Appl. 2014, 160 (2014)

23. Chauhan, S., Karapinar, E.: Some integral type common fixed point theorems satisfying $\Psi$-contractive conditions Bull. Belg. Math. Soc. Simon Stevin 21(4), 593-612 (2014)

24. Geraghty, M.: On contractive mappings. Am. Math. Soc. 40, 604-608 (1973)

25. Kannan, R.: Some results on fixed points-II. Am. Math. Mon. 76(4), 405-408 (1969)

26. Qawaqneh, H., Noorani, M.S., Shatanawi, W., Aydi, H., Alsamir, H.: Fixed point results for multi-valued contractions in b-metric spaces. Mathematics 7(2), 132 (2019)

27. Karapınar, E., Petruşel, A., Petruşel, G.: On admissible hybrid Geraghty contractions. Carpath. J. Math. 36(3), 433-442 (2020)

28. Priyobarta, N., Khomdram, B., Rohen, Y., Saleem, N.: On generalized rational $\alpha$-Geraghty contraction mappings in G-metric spaces. J. Math. 2021, Article ID 6661045 (2021)

29. Karapinar, E., Pitea, A.: On $\alpha-\psi$-Geraghty contraction type mappings on quasi-Branciari metric spaces. J. Nonlinear Convex Anal. 17(7), 1291-1301 (2016)

30. Karapinar, E., Samet, B.: A note on $\psi$-Geraghty type contractions. Fixed Point Theory Appl. 2014, 26 (2014)

31. Afshari, H., Alsulam, H.H., Karapinar, E.: On the extended multivalued Geraghty type contractions. J. Nonlinear Sci. Appl. 9(6), 4695-4706 (2016)

32. Afshari, H., Aydi, H., Karapinar, E.: On generalized $\alpha-\psi$-Geraghty contractions on b-metric spaces. Georgian Math. J. 27, 9-21 (2020)

33. Alqahtani, B., Fulga, A., Karapinar, E.: A short note on the common fixed points of the Geraghty contraction of type $E_{S, T}$. Demonstr. Math. 51, 233-240 (2018)

34. Karapinar, E., Czerwik, S., Aydi, H.: $(\alpha, \psi)$-Meir-Keeler contraction mappings in generalized b-metric spaces. J. Funct. Spaces 2018, Article ID 3264620 (2018)

35. Asadi, M., Karapinar, E., Kumar, A.: $\alpha-\psi$-Geraghty contractions on generalized metric spaces. J. Inequal. Appl. 2014 $423(2014)$

36. Karapinar, E.: A discussion on " $\alpha-\psi$-Geraghty contraction type mappings". Filomat 28(4), 761-766 (2014)

37. Alamgir, N., Kiran, Q., Isik, H., Aydi, H.: Fixed point results via a Hausdorff controlled type metric. Adv. Differ. Equ. 2020, 24 (2020)

38. Bae, J.S., Cho, S.H., Karapinar, E.: Fixed point theorems for $\alpha$-Geraghty contraction type maps in metric spaces. Fixed Point Theory Appl. 2013, 329 (2013)

39. Rus, I.A.: Weakly Picard mappings. Comment. Math. Univ. Carol. 34, 769-773 (1993)

40. Rus, I.A.: Picard operators and applications. Sci. Math. Jpn. 58, 191-219 (2003)

41. Singh, H., Baleanu, D., Srivastava, H.M., Dutta, H., Jhag, N.K.: Solution of multi-dimensional Fredholm equations using Legendre scaling functions. Appl. Numer. Math. 150, 313-324 (2020) 